\title{
Constraints on SN Ia Progenitors and ICM Enrichment from Field and Cluster SN Rates
}

\author{
D. Maoz and A. Gal-Yam
}

School of Physics and Astronomy, Tel Aviv University, Tel Aviv, Israel; dani@wise.tau.ac.il, avishay@wise.tau.ac.il

Summary. The iron mass in galaxy clusters is about 6 times larger than could have been produced by core-collapse $\mathrm{SNe}$, assuming the stars in cluster galaxies formed with a standard IMF. Type-Ia SNe have been proposed as the alternative dominant iron source. We use our HST measurements of the cluster SN-Ia rate at high redshift to study the cluster iron enrichment scenario. The measurements can constrain the star-formation epoch and the SN-Ia progenitor models via the mean delay time between the formation of a stellar population and the explosion of some of its members as SNe-Ia. The low observed rate of cluster SNe-Ia at $z \sim 1$ pushes back the star-formation epoch in clusters to $z>2$, and implies a short delay time. We also show a related analysis for high- $z$ field SNe which implies, under some conditions, a long SN-Ia delay time. Thus, cluster enrichment by core-collapse SNe from a top-heavy IMF may remain the only viable option.

\section{The SN Ia Rate in $z \leq 1$ Galaxy Clusters and the Source of Cluster Iron}

The iron mass in galaxy clusters is about 6 times larger than could have been produced by core-collapse supernovae (SNe), assuming the stars in the cluster formed with a standard initial mass function (IMF; e.g., [10]). SNe Ia have been proposed as the alternative dominant iron source. Different SN Ia progenitor models predict different "delay functions", between the formation of a stellar population and the explosion of some of its members as SNe Ia. We use updated measurements of the total iron mass-to-light ratio in rich clusters to normalize the predicted SN Ia rate in clusters vs. redshift, using the delay function parameterization of [6]. We then use our previous measurements of the cluster SN Ia rate at high redshift [2] to constrain SN Ia progenitor models and the star-formation epoch in clusters. The low observed rate of cluster SNe Ia at $z \sim 0-1$ (Fig. 1) means that, if SNe Ia produced the observed amount of iron, they must have exploded at even higher $z$. This puts a $>95 \%$ upper limit on the mean SN Ia delay time of $\tau<2$ Gyr $(<5 \mathrm{Gyr})$ if the stars in clusters formed at $z_{f}<2\left(z_{f}<3\right)$, assuming $H_{o}=70 \mathrm{~km} \mathrm{~s}^{-1} \mathrm{Mpc}^{-1}$ (see [5] for full details). In the next section, we show that, for some current versions of cosmic (field) star formation history (SFH), observations of field 

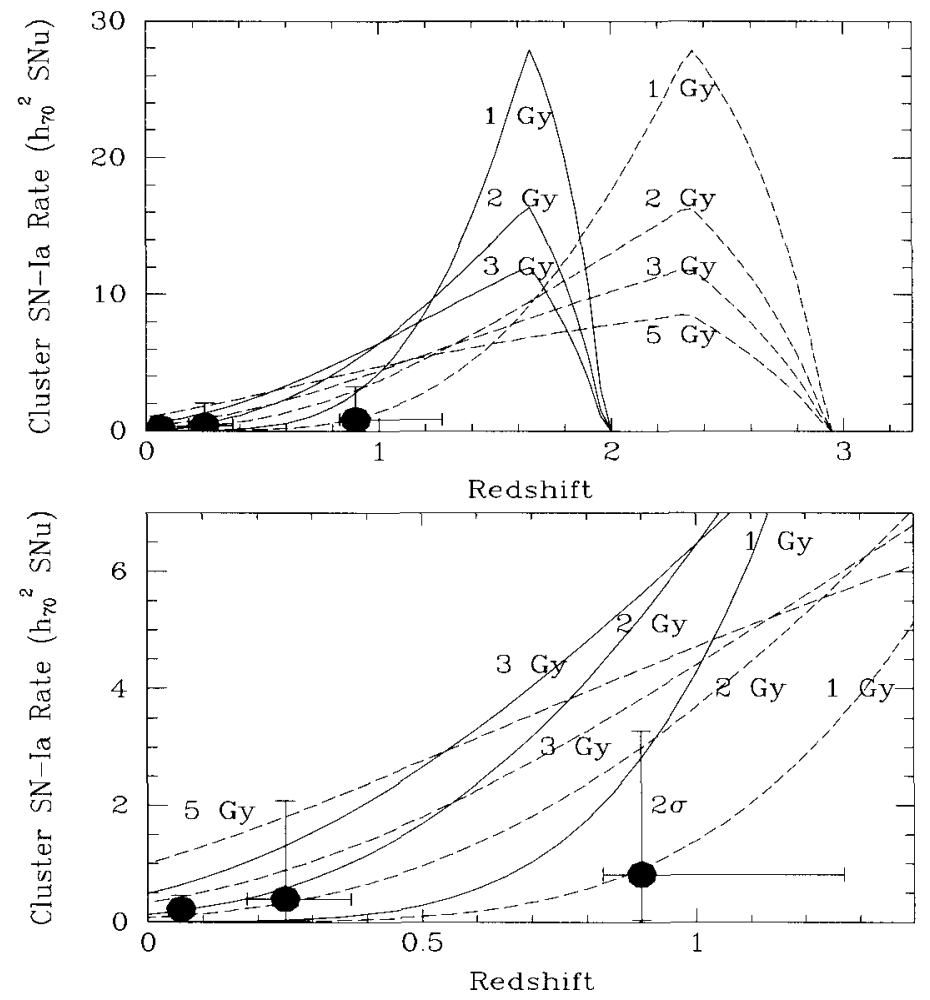

Fig. 1. Predicted SN Ia rates vs redshift, if most of the iron mass in clusters is produced by type-Ia SNe following a brief burst of star formation at redshift $z_{f}=2$ (solid curves) and $z_{f}=3$ (dashed curves). The bottom panel is a zoom on the low $z$ region of the top panel. The different curves are for SN Ia transfer functions with mean delay times, $\tau$, as marked. Cluster SN Ia rate measurements are by $[2,9]$. The latter are shown with $95 \%$-confidence vertical error bars. The horizontal error bars give the visibility-time-weighted redshift ranges of the clust,er samples. The $z_{f}=2$ models (solid curves) with $\tau \geq 2$ Gyr are clearly ruled out by the $z \sim 1$ SN-rate measurement, even after accounting for a $30 \%$ uncertainty in the normalization of the models. The $z_{f}=3$ model (dashed curves) with $\tau=5$ Gyr predicts unacceptably high rates at low $z$.

SNe Ia place a lower bound on the delay time, $\tau>3$ Gyr. If these SFHs are confirmed, the entire range of $\tau$ will be ruled out. Cluster enrichment by core-collapse SNe from a top-heavy IMF will then remain the only viable option. 

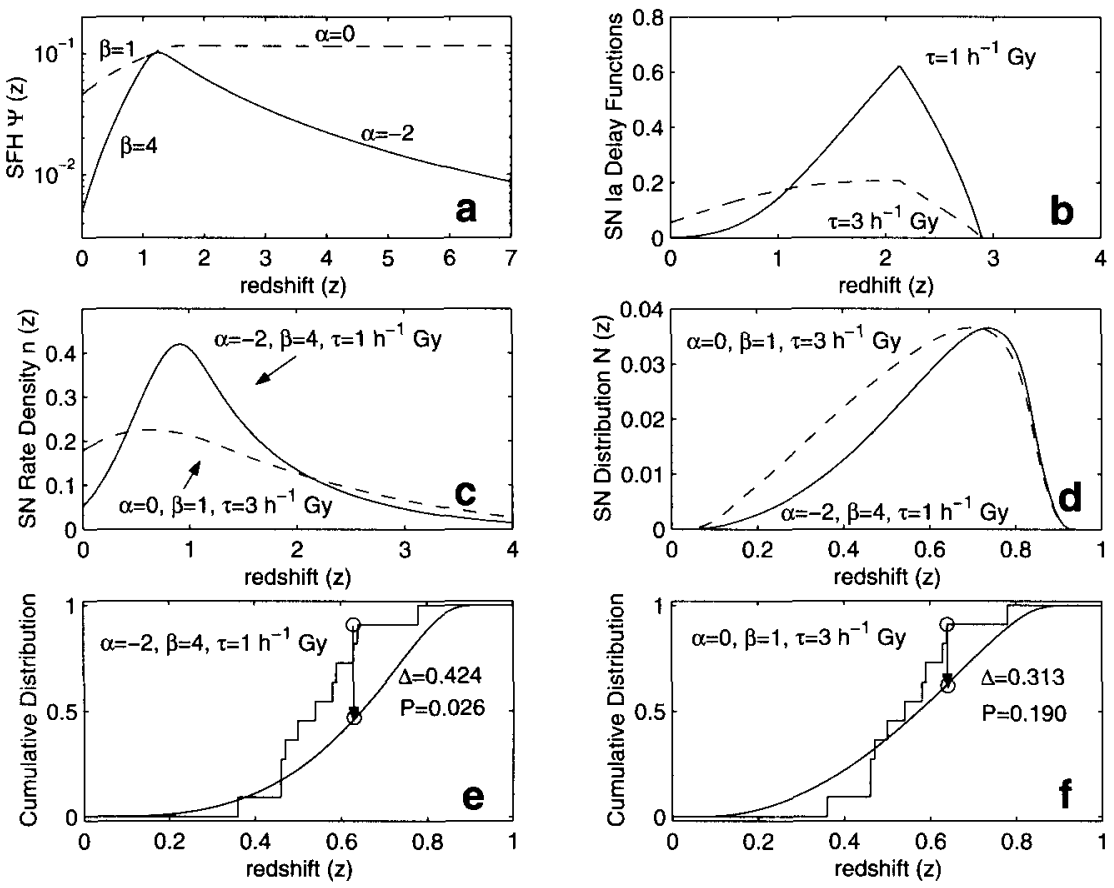

Fig. 2. Illustration of the modeling and comparison to data. The $\mathrm{SFH}(\Psi(z))$ is modeled by two power laws, smoothly joined at $z=1.2$. We denote the high- $z$ index with $\alpha$ and the low- $z$ index with $\beta$. Panel a shows two examples, a "Madau" $\mathrm{SFH}$, with a peak at $z=1.2$ (solid curve), and a shallower model (dashed curve) reflecting the proposed modifications by $[1,11]$. Panel $\mathbf{b}$ shows two examples of the expected SN Ia rate density following a brief burst of star formation. These delay functions are calculated using the prescription of [6], with characteristic exponential delay times of $\tau=1 h^{-1}$ Gyr (solid) and $\tau=3 h^{-1}$ Gyr (dashed). For display purposes, an arbitrary redshift of $z=3$ has been chosen for the burst of star formation. SFH models are convolved with a delay function, and the resulting SN rate densities $n(z)$ for a "Madau" SFH with $\tau=1 h^{-1}$ Gyr (solid) and a "CowieSteidel" SFH with $\tau=3 h^{-1}$ Gyr (dashed) are shown in panel c. Panel $\mathbf{d}$ shows the predicted SN distributions, $N(z)$, for the models of panel $\mathbf{c}$, in a survey with the same observational parameters of the SCP search. KS tests show that the cumulative version of $N(z)$ from a model combining a "Madau" SFH with a typical delay time of $\tau=1 h^{-1} \mathrm{Gyr}$ (panel $\mathbf{e}$ ) is ruled out by the data, while a model with "Cowie-Steidel" SFH and $\tau=3 h^{-1}$ Gyr is consistent with the data (panel f). This "forward modeling" type of analysis is more powerful than the derivation of an observed SN rate at a mean $z$ from the data (e.g., $[8,12]$ ) and comparison to predictions (as in panel c), since no binning over redshift is carried out. Vertical axis units are arbitrary in panels a-d. 

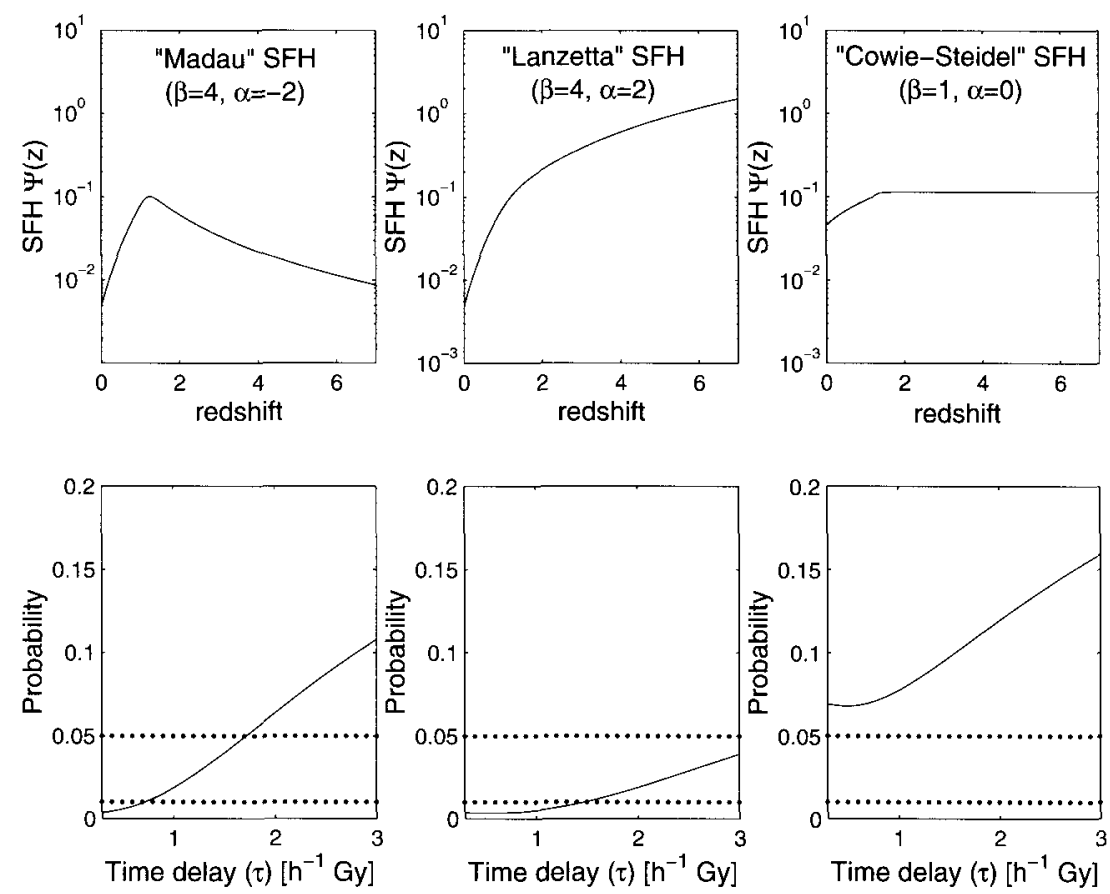

Fig. 3. Probability of SN Ia time delay values $\tau$, given the data, for particular SFH models. Assuming the SFH models shown in the upper panels, we can constrain the allowed values of $\tau$ by the probability derived from the KS test (lower panels). Points below the upper and lower dotted lines are ruled out at $95 \%$ and $99 \%$ confidence, respectively.

\section{The Redshift Distribution of Field SNe Ia: Constraints on Progenitors and Cosmic Star Formation History}

In this section, we use the redshift distribution of SNe Ia discovered by the Supernova Cosmology Project (Pain et al. 2002) to constrain the star formation history (SFH) of the Universe and SN Ia progenitor models. Fig. 2 illustrates how, for a given choice of parameters describing the SFH and SN Ia delay time, we predict the observed cumulative (i.e., unbinned) redshift distribution in a given survey, and compare it to the data. Given some of the recent determinations of the SFH, the observed SN Ia redshift distribution indicates a long ( $~\left(1 h^{-1} \mathrm{Gyr}\right.$ ) mean delay time between the formation of a stellar population and the explosion of some of its members as SNe Ia (Fig. 3). For example, if the Madau et al. (1998) SFH is assumed, the delay time $\tau$ is constrained to be $\tau \geq 1.7(\tau \geq 0.7) h^{-1}$ Gyr at the $95 \%(99 \%)$ confidence level (CL). SFHs that rise at high redshift, similar to those advocated by Lanzetta et al. (2002), are inconsistent with the data at the 95\% CL unless $\tau>3.5 h^{-1}$ 
Gyr. Long time delays disfavor progenitor models such as edge-lit detonation of a white dwarf accreting from a giant donor, and the carbon core ignition of a white dwarf passing the Chandrasekhar mass due to accretion from a subgiant (e.g., [13]). The SN Ia delay may be shorter, thereby relaxing some of these constraints, if the field star formation rate falls, between $z=1$ and the present, less sharply than implied, e.g., by the original Madau plot. The discovery of larger samples of high- $z$ SNe Ia by forthcoming observational projects should yield strong constraints on the progenitor models and the SFH (see [3] for full details). In the previous section we have demonstrated that if SNe Ia produce most of the iron in galaxy clusters, and the stars in clusters formed at $z \sim 2$, the SN Ia delay time must be lower than 2 Gyr. If so, then the Lanzetta et al. [4] SFH is inconsistent with the data presented here (Fig. 3).

\section{Conclusions}

The large mass of iron in clusters, combined with the low SN Ia rate we have measured in $z \sim 1$ clusters, require that, if SNe Ia are the dominant iron source, their delay time must be short. This would cast doubt, e.g., on the double degenerate models. The observed redshift distribution of field SNe Ia implies a slowly changing field SNR(z) for SNe Ia. For several popular starformation histories, this then indicates a long delay time. Taken together, these constraints may suggest that the iron in clusters is from core-collapse $\mathrm{SNe}$, from an early stellar population with a top-heavy IMF.

\section{References}

1. L.L. Cowie, A. Songaila, A.J. Barger: Astron. J. 118, 603 (1999)

2. A. Gal-Yam, D. Maoz, K. Sharon: Mon. Not. R. Astron. Soc. 332, 37 (2002)

3. A. Gal-Yam D. Maoz: Mon. Not. R. Astron. Soc. 347, 942 (2004)

4. K.M. Lanzetta et al. : Astrophys. J. 570, 492 (2002)

5. D. Maoz, A. Gal-Yam: Mon. Not. R. Astron. Soc. 347, 951 (2004)

6. P. Madau, M. Della Valle, N. Panagia: Mon. Not. R. Astron. Soc. 297, L17 (1998)

7. P. Madau et al. : Mon. Not. R. Astron. Soc. 283, 1388 (1998)

8. R. Pain et al. : Astrophys. J. 577, 120 (2002)

9. D. Reiss: The Rate of Supernovae in the Nearby and Distant Universe. Ph.D. Thesis (Univ. Washington: Seattle, 2000)

10. A. Renzini: Astrophys. J. 488, 35 (1997)

11. C.C. Steidel et al. : Astrophys. J. 519, 1 (1999)

12. J.L. Tonry et al. : Astrophys. J. 594, 1 (2003)

13. L.R. Yungelson, M. Livio: Astrophys. J. 528, 108 (2000) 